Pacific Journal of Mathematics

AN APPLICATION OF A FAMILY HOMOTOPY EXTENSION 


\title{
AN APPLICATION OF A FAMILY HOMOTOPY EXTENSION THEOREM TO ANR SPACES
}

\author{
A. H. KRUSe and P. W. Liebnitz
}

The first of the writers, on p. 206 of Introduction to the Theory of Block Assemblages and Related Topics in Topology, NSF Research Report, University of Kansas, 1956, defined a clean-cut pair to be any pair $(X, A)$ in which $X$ is a metrizable space, $A$ is a closed subset of $X, A$ is a strong deformation neighborhood retract of $X$, and $X-A$ is an $A N R$. It is shown in the present paper that for each clean-cut pair $(X, A)$, $X$ is an $A N R$ if and only if $A$ is an $A N R$. A consequence is that for each locally step-finite clean-cut block assemblage (cf. the report cited above), the underlying space is an $A N R$. One of the central tools is a family homotopy extension theorem.

Consider a topological space $X$ and a set $A \subset X$.

Suppose $A \subset N \subset X$. A strong deformation retraction in $X$ of $N$ onto $A$ is a retraction $r$ of $N$ onto $A$ such that there is a homotopy $H: N \times I \rightarrow X$ between the identity map on $N$ and $r$ which leaves $A$ pointwise fixed at each stage. Also, $A$ is a strong deformation retract in $X$ of $N$ if and only if there is a strong deformation retraction in $X$ of $N$ onto $A$. (These definitions are handled more generally in [4, pp. 109-111].) A is a strong deformation neighborhood retract of $X$ if and only if for each neighborhood $U$ of $A$ in $X$ there is a neighborhood $V$ of $A$ in $U$ such that $A$ is a strong deformation neighborhood retract in $U$ of $V$. (This definition is taken from [4, p. 127].) It is observed in [4, pp. 127-128] that $A$ is a strong deformation neighborhood retract of $X$ if and only if $A$ is a strong deformation retract in $X$ of some neighborhood of $A$.

By an $A N R$ we shall mean an $A N R$ relative to the class of all metrizable spaces.

In $[4$, p. 206] the pair $(X, A)$ is defined to be clean-cut if and only if $X$ is metrizable, $A$ is a closed subset of $X, A$ is a strong deformation neighborhood retract of $X$, and $X-A$ is an $A N R$.

In $\S 2$ it will be shown that if $(X, A)$ is a clean-cut pair, then $X$ is an $A N R$ if and only if $A$ is an $A N R$. The "only if" part is trivial. The proof of the "if" part will be based on the usual $L C$ characterization of an $A N R$ and the following proposition from [4, p. 181] (the hypothesis there that $\left\{X_{j}\right\}_{j \in J}$ covers $X$ is inessential since $X$ and $K$ may be added to the respective families).

Proposition 1.1. Suppose that $X$ is a topological space and that

Received October 12, 1964. 
$\left\{X_{j}\right\}_{j \in J}$ is a family of subsets of $X$. Suppose that $K$ is a simplicial complex $\left(|K|\right.$ having the usual $C W$-topology) and that $\left\{K_{j}\right\}_{j \in J}$ is a family of subcomplexes of $K$. Suppose that

$$
f:\left(|K|,\left|K_{j}\right|\right)_{j \in J} \rightarrow\left(X, X_{j}\right)_{j \in J}
$$

is a continuous map, $L$ is a subcomplex of $K$, and

$$
H:\left(|L| \times I,\left|L \cap K_{j}\right| \times I\right)_{j \in J} \rightarrow(X, X)_{j \in J}
$$

is a homotopy from $f|| L \mid$ to some map

$$
g:\left(|L|,\left|L \cap K_{j}\right|\right)_{j \in J} \rightarrow\left(X, X_{j}\right)_{j \in J} \cdot
$$

Then $H$ has an extension

$$
H^{\prime}:\left(|K| \times I,\left|K_{j}\right| \times I\right)_{j \in J} \rightarrow\left(X, X_{j}\right)_{j \in J}
$$

which is a homotopy from $f$ to some extension of $g$.

The reader may read $\S 2$ on the basis of 1.1 and standard results from $A N R$ theory. In [4, p. 181], 1.1 is done with $C W$-complexes in place of simplicial complexes. If the set $J$ in 1.1 is empty, we get one of several homotopy extension theorems. We may call 1.1 a family homotopy extension theorem. For a general treatment of homotopy extension theorems and family homotopy extension theorems, see [4, pp. 210-217].

2. Results for pairs $(X, A)$. Each simplicial complex will have the $C W$-topology. Consider any class $\mathscr{K}$ of simplicial complexes. As in [4, pp. 231-232], $\mathscr{K}$ is admissible if and only if $\mathscr{K}$ is closed under subcomplexes and isomorphic images. Suppose that $X$ is a topological space, $\mathscr{K}$ is an admissible class of simplicial complexes, and $m$ is a nonnegative integer. Then, as in [4, p. 232], $X$ is $L C$ from $m$ upward relative to $\mathscr{K}$ if and only if for each covering $\mathscr{U}$ of $X$ by open subsets of $X$ there is a covering $\mathscr{Y}$ of $X$ by open subsets of $X$ such that $\left(^{*}\right)$ below holds.

$\left(^{*}\right)$ If $K \in \mathscr{K}$, if $L$ is a subcomplex of $K$, if $K^{m} \subset L\left(K^{m}\right.$ is the $m$-skeleton of $K)$, if $g:|L| \rightarrow X$ is a $\mathscr{Y}$-subordinate partial realization of $K$ in $X$ (thus, for each $\sigma \in K, g(\bar{\sigma} \cap|L|) \subset$ some member of $\mathscr{V})$, then $g$ extends to a $\mathscr{U}$-subordinate full realization $f:|K| \rightarrow X$ of $K$ in $X$.

Also, $X$ is $L C$ relative to $\mathscr{K}$ if and only if $X$ is $L C$ from 0 upward relative to $\mathscr{K}$. Also, $X$ is $L C$ if and only if $X$ is $L C$ relative to the class of all simplicial complexes.

The following lemma is probably well-known and follows immediately from standard theorems (e.g., cf. [3, (A), p. 86]). In fact, 
one could replace $I$ by any compact space.

Lemma 2.1. Suppose that $N$ and $X$ are spaces and $A$ is a closed subset of $N$. Suppose that $H: N \times I \rightarrow X$ is continuous. Suppose that $\mathscr{U}$ is a covering of $H(A \times I)$ by open subsets of $X$ and that for each $a \in A, H(\{a\} \times I) \subset U$ for some $U \in \mathscr{Q}$. Then there exists a covering $\mathscr{Y}$ of $A$ by open subsets of $N$ such that for each $V \in \mathscr{Y}, H(V \times I) \subset$ some member of $\mathscr{U}$.

Suppose that $X$ is a space and $\mathscr{Y}^{-}$is a set of subsets of $X$. If $A \subset X$, the star of $A$ with respect to $\mathscr{V}$ is the union of those elements of $\mathscr{V}$ which meet $A$ and will be denoted $\operatorname{St}(A ; \mathscr{V})$. If $\mathscr{U}$ and $\mathscr{V}$ are sets of subsets of $X, \mathscr{Y}$ will be said to star-refine (or *-refine) $\mathscr{U}$ if and only if for each $V \in \mathscr{V}, S t(V ; \mathscr{V})$ is a subset of a member of $\mathscr{U}$. In this case, $\mathscr{Y}$ will be called a star-refinement (or *-refinement) of $\mathscr{U}$.

THEOREM 2.2. Suppose that $X$ is a normal and paracompact space and $A$ is a nonvoid closed subset of $X$ which is a strong deformation neighborhood retract of $X$. Let $\mathscr{K}$ be an admissible class of simplicial complexes, and let $m$ be a nonnegative integer. Suppose that $A$ and $X-A$ are LC from $m$ upward relative to $\mathscr{K}$. Then $X$ is $L C$ from $m$ upward relative to $\mathscr{K}$.

Proof. Consider any covering $\mathscr{U}$ of $X$ by open subsets of $X$. Let $\mathscr{U}^{\prime}$ be a *-refinement of $\mathscr{U}$ by open subsets of $X$ which covers $X$. Let $N$ be an open neighborhood of $A$ in $X$ such that $A$ is a strong deformation retract in $X$ of $N$. Thus there is a homotopy $H: N \times I \rightarrow X$ such that $H(u, t)=u$ for each $u \in A$ and each $t \in I$ and such that $H(u, 0)=u$ and $H(u, 1)=r(u)$ for each $u \in N$, where $r: N \rightarrow A$ is some retraction onto $A$. Let $\mathscr{V}_{1}$ be a covering of $A$ by open subsets of $N$ which refines $\mathscr{U}^{\prime}$ such that if $K \in \mathscr{K}$, if $L$ is a subcomplex of $K$, if $K^{m} \subset L$, if $g:|L| \rightarrow A$ is a partial realization of $K$ in $A$ subordinate to $\mathscr{V}_{1}$, then $g$ can be extended to a full realization of $K$ in $A$ subordinate to $\mathscr{U}^{\prime}$. Using 2.1, let $\mathscr{V}_{2}$ be a covering of $A$ by open subsets of $N$ such that for each $V \in \mathscr{V}_{2}, H(V \times I) \subset$ some member of $\mathscr{V}_{1}$. Observe that $\mathscr{V}_{2}$ refines $\mathscr{V}_{1}$. Let $\mathscr{V}_{3}$ be a *-refinement of $\mathscr{V}_{2}$ by open subsets of $N$ which covers $A$. Let $\mathscr{Y}_{A}$ be a refinement of $\mathscr{V}_{3}$ by open subsets of $N$ which covers $A$. Let $N_{3}=\cup \mathscr{V}_{3}$ and $N_{A}=\cup \mathscr{V}_{A}$. We may and do require that $\bar{N}_{A} \subset N_{3}$. Let $\mathscr{W}_{1}$ be a covering of $X-\bar{N}_{A}$ by open subsets of $X-\bar{N}_{A}$ which refines $\mathscr{U}^{\prime}$. Let $\mathscr{W}=\mathscr{V}_{3} \cup \mathscr{W}_{1}$. Let $\mathscr{V}_{x_{-A}}$ be an open covering of $X-A$ such that if $K \in \mathscr{K}$, if $L$ is a subcomplex of $K$, if $K^{m} \subset L$, if $g:|L| \rightarrow X-A$ is a partial realization of $K$ in $X-A$ subordinate to $\mathscr{Y}_{X-A}$, then $g$ can be extended to a full realization of $K$ in $X-A$ 
subordinate to $\mathscr{W} . \mathscr{V}=\mathscr{Y}_{A} \cup \mathscr{Y}_{X-A} ; \mathscr{Y}$ is a covering of $X$ by open subsets of $X$.

Now consider $K \in \mathscr{K}$. Consider any partial realization $\alpha:|L| \rightarrow X$ of $K$ in $X$ subordinate to $\mathscr{V}$ such that $K^{m} \subset L$. Define

$$
\begin{aligned}
K_{A} & =\left\{\sigma \in K: \alpha(\bar{\sigma} \cap|L|) \subset \text { some member of } \mathscr{V}_{A}\right\}, \\
K_{X-A} & =\left\{\sigma \in K: \alpha(\bar{\sigma} \cap|L|) \subset \text { some member of } \mathscr{V}_{X-A}\right\} .
\end{aligned}
$$

$K_{A}$ and $K_{X-A}$ are subcomplexes of $K$, and $K_{A} \cup K_{X-A}=K$.

Now $\alpha|| K_{X-A} \cap L|:| K_{X-A} \cap L \mid \rightarrow X-A$ is a partial realization of $K_{X-A}$ in $X-A$ subordinate to $\mathscr{V}_{X-A}$, and $\left(K_{X_{-A}}\right)^{m} \subset K_{X-A} \cap L$. Hence $\alpha|| K_{X-A} \cap L \mid$ extends to a full realization $\beta:\left|K_{X-A}\right| \rightarrow X-A$ of $K_{X-A}$ in $X-A$ subordinate to $\mathscr{W}$.

Define $\hat{\beta}:\left|K_{X-A} \cup L\right| \rightarrow X$ by

$$
\widehat{\beta} \mid \bar{\sigma}=\left\{\begin{array}{l}
\alpha \mid \bar{\sigma} \text { if } \sigma \in L, \\
\beta \mid \bar{\sigma} \text { if } \sigma \in K_{X-A} .
\end{array}\right.
$$

$\hat{\beta}$ is obviously continuous.

Set $M=K_{\Delta} \cap\left(K_{x-\Delta} \cup L\right)$ and $\widetilde{\beta}=\widehat{\beta}|| M \mid$. Consider $\sigma \in K_{\Delta}$. Now $\widetilde{\beta}(\bar{\sigma} \cap|L|) \subset V$ for some $V \in \mathscr{V}_{A}$. Consider a face $\tau \in K_{X-4}$ of $\sigma$. Now $\widehat{\beta}(\bar{\tau}) \subset W$ for some $W \in \mathscr{W}$. Since also $\widehat{\beta}(\bar{\tau} \cap|L|)=\alpha(\bar{\tau} \cap|L|) \subset$ $\alpha(\bar{\sigma} \cap|L|) \subset$ some member of $\mathscr{Y}_{A}, W \cap N_{A}$ is nonvoid. Hence $W \in \mathscr{V}_{3}$. It follows (since also $\mathscr{V}_{4}$ refines $\mathscr{V}_{3}$ and $\mathscr{V}_{3} *$-refines $\mathscr{V}_{2}$ ) that

$$
\widetilde{\beta}\left(\bar{\sigma} \cap\left|K_{X-A} \cup L\right|\right) \subset S t\left(V ; \mathscr{V}_{3}\right) \subset \text { some member of } \mathscr{V}_{2} \text {. }
$$

Thus $\widetilde{\beta}:|M| \rightarrow N$ is a partial realization of $K_{\Delta}$ in $N$ subordinate to $\mathscr{V}_{2}$.

For each $\sigma \in K_{A}, H(\widetilde{\beta}(\bar{\sigma} \cap|M|) \times I) \subset H\left(V_{2} \times I\right) \subset V_{1}$ for some $V_{2} \in \mathscr{V}_{2}, V_{1} \in \mathscr{V}_{1}$. For each $u \in|M|$ and each $t \in I$ put

$$
G_{t}(u)=G(u, t)=H(\widetilde{\beta}(u), t),
$$

and observe that $G_{1}(u)=r(\widetilde{\beta}(u)) \in A$ for each $u \in|M|$. Thus $G_{1}:|M| \rightarrow A$ is a partial realization of $K_{A}$ in $A$ subordinate to $\mathscr{V}_{1}$. Hence $G_{1}:|M| \rightarrow A$ extends to a full realization $J_{1}:\left|K_{A}\right| \rightarrow A$ subordinate to $\mathscr{U}^{\prime}$. Consider $\sigma \in K_{\Delta}$. We have $J_{1}(\bar{\sigma}) \subset U^{\prime}$ for some $U^{\prime} \in \mathscr{U}^{\prime}$. Also $G((|M| \cap \bar{\sigma}) \times I) \subset V$ for some $V \in \mathscr{\mathscr { V }}$. Hence $G_{1}(|M| \cap \bar{\sigma}) \subset V$. Hence $J_{1}(\bar{\sigma}) \subset S t\left(U^{\prime} ; \mathscr{U}^{\prime}\right) \subset U_{\sigma}$ for some $U_{\sigma} \in \mathscr{\mathscr { C }}$ and likewise $G((|M| \cap \bar{\sigma}) \times I) \subset U_{\sigma}$. Thus

$$
G:(|M| \times I,(|M| \cap \bar{\sigma}) \times I)_{\sigma \in K_{A}} \rightarrow\left(X, U_{\sigma}\right)_{\sigma \in K_{A}}
$$

is a homotopy from

$$
\left.G_{0}:(|M|,|M| \cap \bar{\sigma})\right)_{\sigma \in K_{A}} \rightarrow\left(X, U_{\sigma}\right)_{\sigma \in K_{A}}
$$




$$
J_{1}|| M \mid:(|M| \cap \bar{\sigma})_{\sigma \in K_{A}} \rightarrow\left(X, U_{\sigma}\right)_{\sigma \in K_{A}} .
$$

By 1.1, $G$ extends to

$$
G^{\prime}:\left(\left|K_{\Delta}\right| \times I, \bar{\sigma} \times I\right)_{\sigma \in K_{A}} \rightarrow\left(X, U_{\sigma}\right)_{\sigma \in K_{A}}
$$

which is a homotopy from an extension $G_{0}^{\prime}$ of $G_{0}$ to $J_{1}$.

Define $\phi:|K| \rightarrow X$ by

$$
\phi \mid \bar{\sigma}=\left\{\begin{array}{l}
\beta \mid \bar{\sigma} \text { if } \sigma \in K_{X-A}, \\
G_{0}^{\prime} \mid \bar{\sigma} \text { if } \sigma \in K_{\Delta} .
\end{array}\right.
$$

It is easily seen that $\phi$ is a full realization of $K$ in $X$ subordinate to $\mathscr{U}$ which extends $\alpha:|L| \rightarrow X$.

The proof is complete.

For another interesting application of 1.1, cf. the proof of [4, 4.3 , p. 249], the application occurring in [4, p. 251]. The theorem proved there is used to characterize a metric space being $\mathscr{C}$-dominated by simplicial complexes (for open coverings $\mathscr{C}$ ) in terms of $L C$ properties. See also $[4,3.6$, p. 277].

Theorem 2.3. Suppose that $(X, A)$ is a clean-cut pair. Then $X$ is an $A N R$ if and only if $A$ is an $A N R$.

Proof. If $X$ is an $A N R$, then so is the closed neighborhood retract $A$ of $X$ by standard $A N R$ theory (also, cf. [4, 1.3, p. 206]). Suppose now that $A$ is an $A N R$. By [4, 3.2, p. 275] or $[1, \mathrm{p} .364]$, a metrizable space is an $A N R$ if and only if it is $L C$. Hence $X-A$ and $A$ are $L C$. By 2.2, $X$ is $L C$. Thus $X$ is an $A N R$.

3. Results for clean-cut block assemblages. The definitions pertinent to this section are too long to be given here and may be found in [4, p. 70] (for block assemblage), [4, p. 94] (for locally stepfinite), and [4, p. 207] (for clean-cut applied to block assemblages). We remark here only that clean-cut block assemblage is essentially a generalization of $C W$-complex, suitably embedded Euclidean cells being replaced by suitably embedded $A N R$.

THEOREM 3.1. Suppose that $(X, \mathscr{B})$ is a locally step-finite clean-cut block assemblage. Then $X$ is an ANR.

Proof. The notation of $[4$, p. 70$]$ will be used. By [4, 8.6, p. 98], $X$ is metrizable. It suffices to show that $S_{\mu}$ is an $A N R$ for each $\mu \leqq \nu$. Assume the contray. Thus we have some $\mu \leqq \nu$ with $S_{\mu}$ not an $A N R$ and with $S_{\lambda}$ an $A N R$ for each $\lambda<\mu$. If $\mu=\gamma+1$, then 
$S_{\gamma}$ is an $A N R$ and $S_{\mu}=\left(B_{\mu}-S_{\gamma}\right) \cup S_{\gamma}$ is an $A N R$ by [4, p. 207] and 2.3, contradiction. Hence $\mu$ has no immediate predecessor. Each point of $S_{\mu}$ has $S_{\lambda}$ for a neighborhood in $S_{\mu}$ for some $\lambda<\mu$ (cf. [4, p. 94]). Hence $S_{\mu}$ is locally $A N R$. Hence $S_{\mu}$ is an $A N R$ by $[2,19.2$ or 19.3, p. 341].

Corollary 3.2. Suppose that $(X, \mathscr{B})$ is a clean-cut block assemblage with only finitely many blocks. Then $X$ is an ANR.

\section{REFERENCES}

1. J. Dugundji, An extension of Tietze's theorem, Pacific J. Math. 1 (1951), 353-367.

2. O. Hanner, Retraction and extension of mappings of metric and non-metric spaces, Arkiv für Mathematik, 2 (1952), 315-360.

3. W. Hurewicz and H. Wallman, Dimension Theory, Princeton University Press, Princeton, New Jersey, 1941.

4. A. H. Kruse, Introduction to the Theory of Block Assemblages and Related Topics in Topology, NSF Research Project on Geometry of Function Space, Report No. 6, University of Kansas, Lawrence, Kansas, 1956.

New Mexico State University

AND

UNIVERSITY OF MISSOURI AT KANSAS CITY 


\section{PACIFIC JOURNAL OF MATHEMATICS}

\section{EDITORS}

\section{H. SAMELSON}

Stanford University

Stanford, California

\section{R. M. BLUMenthaL \\ University of Washington \\ Seattle, Washington 98105}

*J. DugundjI

University of Southern California Los Angeles, California 90007

\section{RICHARD ARENS}

University of California

Los Angeles, California 90024

\section{ASSOCIATE EDITORS}
E. F. BECKENBACH
B. H. NeUManN
F. WOLF
K. YosIDA

\section{SUPPORTING INSTITUTIONS}

\author{
UNIVERSITY OF BRITISH COLUMBIA \\ CALIFORNIA INSTITUTE OF TECHNOLOGY \\ UNIVERSITY OF CALIFORNIA \\ MONTANA STATE UNIVERSITY \\ UNIVERSITY OF NEVADA \\ NEW MEXICO STATE UNIVERSITY \\ OREGON STATE UNIVERSITY \\ UNIVERSITY OF OREGON \\ OSAKA UNIVERSITY \\ UNIVERSITY OF SOUTHERN CALIFORNIA
}

\author{
STANFORD UNIVERSITY \\ UNIVERSITY OF TOKYO \\ UNIVERSITY OF UTAH \\ WASHINGTON STATE UNIVERSITY \\ UNIVERSITY OF WASHINGTON \\ AMERICAN MATHEMATICAL SOCIETY \\ CHEVRON RESEARCH CORPORATION \\ TRW SYSTEMS \\ NAVAL ORDNANCE TEST STATION
}

Mathematical papers intended for publication in the Pacific Journal of Mathematics should be typewritten (double spaced). The first paragraph or two must be capable of being used separately as a synopsis of the entire paper. It should not contain references to the bibliography. Manuscripts may be sent to any one of the four editors. All other communications to the editors should be addressed to the managing editor, Richard Arens at the University of California, Los Angeles, California 90024.

50 reprints per author of each article are furnished free of charge; additional copies may be obtained at cost in multiples of 50 .

The Pacific Journal of Mathematics is published monthly. Effective with Volume 16 the price per volume (3 numbers) is $\$ 8.00$; single issues, $\$ 3.00$. Special price for current issues to individual faculty members of supporting institutions and to individual members of the American Mathematical Society: $\$ 4.00$ per volume; single issues $\$ 1.50$. Back numbers are available.

Subscriptions, orders for back numbers, and changes of address should be sent to Pacific Journal of Mathematics, 103 Highland Boulevard, Berkeley 8, California.

Printed at Kokusai Bunken Insatsusha (International Academic Printing Co., Ltd.), No. 6, 2-chome, Fujimi-cho, Chiyoda-ku, Tokyo, Japan.

PUBLISHED BY PACIFIC JOURNAL OF MATHEMATICS, A NON-PROFIT CORPORATION

The Supporting Institutions listed above contribute to the cost of publication of this Journal, but they are not owners or publishers and have no responsibility for its content or policies.

* Paul A. White, Acting Editor until J. Dugundji returns. 


\section{Pacific Journal of Mathematics}

\section{Vol. 16, No. 2 December, 1966}

Loren N. Argabright, Invariant means on topological semigroups ........ 193

William Arveson, A theorem on the action of abelian unitary groups ...... 205

John Spurgeon Bradley, Adjoint quasi-differential operators of Euler

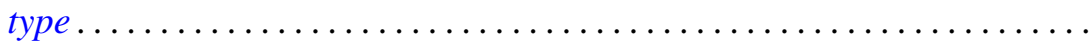

Don Deckard and Lincoln Kearney Durst, Unique factorization in power series rings and semigroups ........................... 239

Allen Devinatz, The deficiency index of ordinary self-adjoint differential operators..................................... 243

Robert E. Edwards, Operators commuting with translations ............ 259

Avner Friedman, Differentiability of solutions of ordinary differential equations in Hilbert space .............................. 267

Boris Garfinkel and Gregory Thomas McAllister, Jr., Singularities in a variational problem with an inequality ......................

Seymour Ginsburg and Edwin Spanier, Semigroups, Presburger formulas,

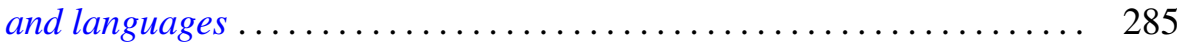

Burrell Washington Helton, Integral equations and product integrals . . . . . . 297

Edgar J. Howard, First and second category Abelian groups with the n-adic topology.........................................

Arthur H. Kruse and Paul William Liebnitz, Jr., An application of a family homotopy extension theorem to ANR spaces.

Albert Marden, I. Richards and Burton Rodin, On the regions bounded by homotopic curves

Willard Miller, Jr., A branching law for the symplectic groups ...

Marc Aristide Rieffel, A characterization of the group algebras of the finite

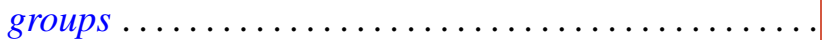

P. P. Saworotnow, On two-sided $H^{*}$-algebras

John Griggs Thompson, Factorizations of p-solvable groups ...

Shih-hsiung Tung, Harnack's inequalities on the classical Cartan

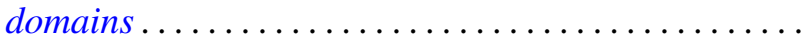

